\title{
Can vibratory feedback be used to improve postural stability in persons with transtibial limb loss?
}

\author{
David Rusaw, PhD; ${ }^{1-2^{*}}$ Kerstin Hagberg, PhD; ${ }^{1}$ Lee Nolan, PhD; ${ }^{2-3}$ Nerrolyn Ramstrand, $\mathbf{P h D}{ }^{\mathbf{2}}$ \\ ${ }^{1}$ Institute for Clinical Sciences, Department of Orthopaedics, Sahlgrenska Academy, University of Gothenburg, Gothenburg, \\ Sweden; ${ }^{2}$ Department of Rehabilitation, School of Health Sciences, Jönköping University, Jönköping, Sweden; \\ ${ }^{3}$ Laboratory for Biomechanics and Motor Control, Department of Neuroscience, Karolinska Institutet and GIH, Stock- \\ holm, Sweden
}

\begin{abstract}
The use of vibration as a feedback modality to convey motion of the body has been shown to improve measures of postural stability in some groups of patients. Because individuals using transtibial prostheses lack sensation distal to the amputation, vibratory feedback could possibly be used to improve their postural stability. The current investigation provided transtibial prosthesis users ( $n=24$, mean age $48 \mathrm{yr}$ ) with vibratory feedback proportional to the signal received from force transducers located under the prosthetic foot. Postural stability was evaluated by measuring center of pressure (CoP) movement, limits of stability, and rhythmic weight shift while participants stood on a force platform capable of rotations in the pitch plane (toes up/toes down). The results showed that the vibratory feedback increased the mediolateral displacement amplitude of CoP in standing balance and reduced the response time to rapid voluntary movements of the center of gravity. The results suggest that the use of vibratory feedback in an experimental setting leads to improvements in fast open-loop mechanisms of postural control in transtibial prosthesis users.
\end{abstract}

Key words: amputation, balance, center of pressure, feedback, limits of stability, postural stability, prosthesis, rhythmic weight shift, transtibial, vibration.

\section{INTRODUCTION}

Sensory feedback from the lower limbs is important for the maintenance of upright gait in humans. The feed- back we receive from various receptors allows us to maintain balance, ambulate and, more importantly, adapt to the physical environment we move in [1-2]. Among persons with lower-limb loss, balance has been shown to be a strong indicator of physical capacity linked to walking ability [3]. Prosthesis users have further been shown to have increased risk of falling, with roughly 52 percent reporting a fall in the previous 12 mo [4]. They also

\footnotetext{
Abbreviations: $\mathrm{AE}=$ angle of excursion, $\mathrm{AMP}_{\mathrm{AP}}=$ anteroposterior sway amplitude, $\mathrm{AMP}_{\mathrm{ML}}=$ mediolateral sway amplitude, $\mathrm{AV}=$ on-axis velocity, $\mathrm{CoG}=$ center of gravity, $\mathrm{CoP}=$ center of pressure, DC = directional control, EMG = electromyography, ICC = intraclass correlation coefficient, LOS = limits of stability, MANOVA = multivariate analysis of variance, $\mathrm{ME}=$ maximum excursion, $\mathrm{MV}_{\mathrm{AP}}=$ anteroposterior velocity, $\mathrm{MV}_{\mathrm{ML}}=$ mediolateral velocity, NOVIB $=$ no vibration (condition), $\mathrm{P}_{\mathrm{AP}}=$ anteroposterior path length, $\mathrm{P}_{\mathrm{ML}}=$ mediolateral path length, PPS = path length per second, RMS = root-mean-square, $\mathrm{RMS}_{\mathrm{AP}}=$ anteroposterior RMS, $\mathrm{RMS}_{\mathrm{ML}}=$ mediolateral RMS, RT = reaction time, RWS = rhythmic weight shift, $\mathrm{SB}=$ standing balance, $\mathrm{SD}=$ standard deviation, $\mathrm{VIB}=$ vibration (condition).

*Address all correspondence to David Rusaw, PhD; Department of Rehabilitation, School of Health Sciences, Jönköping University, Box 1026, 55111, Jönköping, Sweden; +46(0)36-10-12-75; fax: +46(0)36-10-11-80.

Email: david.rusaw@hhj.hj.se http://dx.doi.org/10.1682/JRRD.2011.05.0088
} 
report an increased fear of falling [5] and reduced social participation because of this fear [6]. In persons with a unilateral transtibial amputation due to trauma or vascular disease, poor sensation has been identified not only in the side of the amputation but also in the contralateral foot and knee [7]. This has been identified as another factor contributing to decreased balance and safety [8]. With insufficient feedback from the sensory receptors in the lower limbs, ambulation becomes slower and more cognitively demanding for persons with amputation [9].

Assessment of balance has been shown to identify individuals at risk of falling [10-12]. In individuals over age 65 , increased mediolateral velocity $\left(\mathrm{MV}_{\mathrm{ML}}\right)$ of the center of pressure (CoP), increased mean amplitude of mediolateral movement of the $\mathrm{CoP}$, and increased rootmean-square (RMS) of the mediolateral displacement all have strong associations with future falls [11-12]. Additionally, path length per second (PPS) has been shown to provide the most consistency in differences on multiple testing occasions when nondisabled individuals, patients with Parkinson disease, and geriatric patients were compared [13].

When compared with nondisabled controls, persons with unilateral lower-limb loss have been shown to have increased $\mathrm{CoP}$ excursion in the mediolateral and anteroposterior directions [14] and an increased RMS of the mediolateral and anteroposterior velocities of the CoP [15]. Among individuals with amputation due to vascular disease, performance of standing balance (SB) has been shown to be less stable than among those with limb loss due to trauma [16] as well as nondisabled controls. When the support surface is not stationary or the balance task becomes more complicated, increased measures of instability have been observed in the anteroposterior direction $[14,17]$. Control in the mediolateral and anteroposterior planes is mediated by different joints [18], with stability in the anteroposterior plane largely mediated by the ankle [19]. The decreased stability in the anteroposterior plane is functionally significant because a prosthesis user lacks the sensorimotor control of a physiological ankle in the sagittal plane. Unilateral prosthesis users have asymmetric weight-bearing and shift their body mass toward the intact limb during quiet stance [20]. This asymmetry increases if the support surface is unstable, which is thought to be due to limitations imposed by the prosthetic foot/ankle [17]. Two differing theories exist regarding the way a prosthesis contributes to stability during gait. The first theory states that a rigid prosthetic forefoot keel pro- vides an external torque to the knee joint, which acts to keep it stable [21-22]. In this theory, the stability of the knee relies less on the internal torque provided by the knee muscle extensors. A recent study suggests this may also apply to postural stability; a positive correlation was identified between increased stiffness of a prosthetic foot and increased dynamic balance control, defined as the ratio of ankle torque in response to sway between the intact and prosthetic limbs [23]. The second theory suggests that stability is facilitated by the prosthetic foot's ability to accommodate to uneven surfaces by maintaining contact with the floor for a longer period of time [24]. This theory is supported by Hafner et al. [25], who suggested that the perception of stability was influenced by the ability to extend the amount of time spent in midstance without heel off. Anteroposterior postural stability for prosthetic users is of interest because they perform worse than nondisabled individuals in this plane and the role of the prosthetic foot can be questioned. Tests such as limits of stability (LOS) and rhythmic weight shift (RWS) investigate multiple-plane voluntary control and assessments including anteroposterior movement may explain how transtibial prosthesis users incorporate the prosthesis into the sensorimotor system. To date, prosthetic users have not been investigated using these tests. Because they assess voluntary control of postural stability and demand increased range of motion of the prosthetic foot, the execution of these tests is warranted.

The application of vibrotactile feedback through externally mounted vibrating units (tactors) has been shown to improve specific measures of balance in a number of groups [26-28]. Vibration applied to the trunk of patients with vestibular loss has been demonstrated to reduce measures of postural instability (RMS of CoP) during angular support surface perturbations [28] and multidirectional planar perturbations (RMS of body tilt) [27]. Though less in magnitude, similar patterns were found in a nondisabled sample (RMS of body tilt) [26]. During upright standing, the body behaves similar to an inverted pendulum [19]. It is subsequently possible that the CoP could be an equally beneficial feedback source. This was investigated by Sienko et al. [27], who used vibration as a modality to express information about CoP movement in a sample of persons with vestibular loss and found similar results [27].

Investigations on prosthetic users have indicated that weight distribution and gait symmetry can be improved by utilizing interventions applied unilaterally on the side with 
the prosthesis. These have included the use of electrical feedback [29], vibratory posteffects [30], and feedback via pneumatic air balloons [31-32]. It is not known whether a vibratory stimulus conveying information about pressure from under a prosthetic foot would influence additional measures of postural stability, such as movement of the CoP, LOS, and RWS.

The aim of this study was to evaluate the effects of a vibratory feedback system, applied to the prosthetic users' affected side, on static and dynamic balance in persons with unilateral transtibial limb loss. Three tests were conducted to accomplish this aim: SB, LOS, and RWS. The primary analysis focused on comparison of the main effect of vibration (VIB) versus no vibration (NOVIB) conditions. The experimental hypothesis was that participants would have improved performance in the observed dependent variables when vibratory feedback was available.

\section{METHODS}

\section{Participants}

A total of 24 persons with unilateral transtibial amputation were recruited to participate. Subjects had a mean age of $48 \mathrm{yr}$ (standard deviation [SD] 14), height of 1.77 $\mathrm{m}$ (SD 0.08), and mass of $80 \mathrm{~kg}$ (SD 14). Characteristics of the participants are provided in Table 1. Participants were recruited from five prosthetic clinics in southern Sweden. They were included on the basis that they had no concomitant health issues (such as diabetes, neurological diseases, or peripheral vascular disease), had no current issues regarding fit or function of their current prosthesis (including wounds, blisters, or skin breakdown), and had been a prosthesis user for a period not less than 1 yr. Participants used their currently functioning prosthesis during all data collection. All participants

Table 1.

Participant characteristics.

\begin{tabular}{|c|c|c|c|c|c|c|c|}
\hline Participant & $\begin{array}{c}\text { Cause of } \\
\text { Amputation }\end{array}$ & Sex & $\begin{array}{c}\text { Time since } \\
\text { Amputation (yr) }\end{array}$ & Height (m) & Mass (kg) & Age (yr) & $\begin{array}{l}\text { Residual Limb } \\
\text { Length }^{*}\end{array}$ \\
\hline $\mathrm{A}$ & Trauma & $\mathrm{M}$ & 8 & 1.78 & 73 & 46 & Ordinary \\
\hline B & Infection & M & 5 & 1.8 & 67 & 27 & Ordinary \\
\hline $\mathrm{C}$ & Trauma & M & 4 & 1.9 & 78 & 40 & Ordinary \\
\hline $\mathrm{D}$ & Trauma & $\mathrm{M}$ & 12 & 1.84 & 97 & 65 & Long \\
\hline $\mathrm{E}$ & Trauma & $\mathrm{M}$ & 3 & 1.86 & 82 & 53 & Ordinary \\
\hline G & Trauma & $\mathrm{M}$ & 4 & 1.76 & 100 & 53 & Long \\
\hline $\mathrm{H}$ & Trauma & $\mathrm{F}$ & 33 & 1.59 & 64 & 60 & Long \\
\hline I & Trauma & $\mathrm{M}$ & 2 & 1.78 & 87 & 51 & Ordinary \\
\hline $\mathrm{J}$ & Trauma & $\mathrm{M}$ & 19 & 1.8 & 83 & 33 & Ordinary \\
\hline $\mathrm{K}$ & Trauma & M & 34 & 1.78 & 85 & 47 & Ordinary \\
\hline $\mathrm{O}$ & Osteosarcoma & $\mathrm{F}$ & 28 & 1.71 & 73 & 47 & Long \\
\hline $\mathrm{P}$ & Trauma & $\mathrm{M}$ & 12 & 1.8 & 112 & 26 & Long \\
\hline $\mathrm{Q}$ & Trauma & M & 21 & 1.78 & 81 & 37 & Long \\
\hline $\mathrm{R}$ & Congenital & $\mathrm{F}$ & 25 & 1.54 & 64 & 25 & Long \\
\hline S & Trauma & $\mathrm{M}$ & 13 & 1.8 & 78 & 60 & Ordinary \\
\hline $\mathrm{T}$ & Infection & $\mathrm{F}$ & 5 & 1.82 & 57 & 30 & Ordinary \\
\hline $\mathrm{U}$ & Trauma & $\mathrm{M}$ & 19 & 1.76 & 101 & 62 & Long \\
\hline $\mathrm{V}$ & Congenital & $\mathrm{F}$ & 45 & 1.64 & 64 & 45 & Long \\
\hline W & Thrombosis & M & 8 & 1.79 & 60 & 56 & Long \\
\hline$X$ & Trauma & M & 3 & 1.85 & 88 & 51 & Long \\
\hline
\end{tabular}


had a composite-fiber socket and a gel liner. Suspension and prosthetic feet varied (suspension: locking liner $=17$, sleeve $=3$, elevated vacuum $=4$; prosthetic feet: energy storage and return $=20$, multiaxis $=3$, single-axis $=1$, according to the classification described by Hafner [33]). Residual limb length was classified according to Persson and Liedberg [34] (Table 1).

\section{Equipment}

\section{Force Platform}

Balance tests were conducted using a Pro Balance Master (NeuroCom International Inc; Clackamas, Oregon). This system has been described in previous work and incorporates a $46 \times 46 \mathrm{~cm}$ force plate that is capable of rotating about a single mechanical axis located at the ankles. The rotation occurs in the sagittal plane and corresponds to a toes-up and toes-down movement [35-37]. All force platform data was captured at $100 \mathrm{~Hz}$.

\section{Feedback System}

During experimental trials, participants wore a custom-made device transmitting real-time proportional feedback from pressure sensors located under the prosthetic foot to vibrating tactors located proximally on the thigh (Figure). Four individual Flexiforce transducers (Tekscan, Inc; Boston, Massachusetts) were positioned under the prosthetic foot in anterior, posterior, medial, and lateral positions (Figure). Pressure applied to the force transducers produced a signal that was transmitted to a 4-channel controller with on-board microprocessor. This controller received the voltage output and produced a sine wave signal $(230 \mathrm{~Hz})$ that provided the current to power amplifier modules and individual tactors located on the thigh of the participants (C-2 Tactors, Engineering Acoustics Inc; Casselberry, Florida). Each channel was independent and capable of producing an output of $350 \mathrm{~mA}$ RMS at $250 \mathrm{~Hz}$ to power individual tactors.

\section{Procedure}

Three specific tests of balance were investigated: SB, LOS, and RWS. The repeatability of each of these experimental conditions has been established in previous works: SB = moderate to excellent [38], LOS = moderate to excellent intraclass correlation coefficient (ICC) 0.78 0.93 [39-40] (generalizability coefficient $=0.69-0.91$ ) [41], and RWS = moderate ICC 0.6-0.7 [42]. A power calculation using anteroposterior path length $\left(\mathrm{P}_{\mathrm{AP}}\right)$ of the
CoP from a previous study [17] established that a minimum sample size of $n=24$ was required to detect a statistically significant difference $(p<0.05)$ between two paired groups (prosthesis users vs nondisabled), given a statistical power of 0.8 and a true difference between groups of $1.00 \mathrm{~m} / 20 \mathrm{~s}$.

\section{Test One: Standing Balance}

During the SB test, participants were requested to maintain an upright posture for a period of $20 \mathrm{~s}$ under four sensory conditions: stable support surface with eyes open (condition 1), stable support surface with eyes closed (condition 2), sway-referenced support surface with eyes open (condition 3), and sway-referenced support surface with eyes closed (condition 4). Sway-referencing is a method of delivering inaccurate proprioceptive information about support-surface position [43]. This was accomplished internally using the force-platform software to match the anteroposterior sway excursion angle with a support-surface inclination of the same magnitude. Each condition was repeated three times and data were averaged

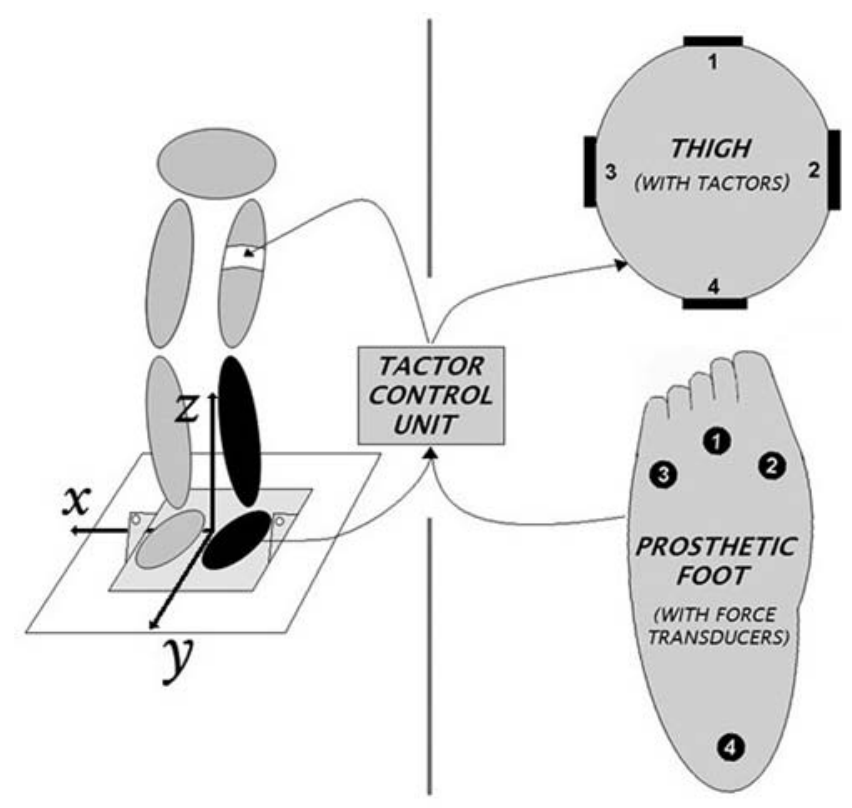

Figure.

Tactor control unit and channel descriptions (1: anterior, 2: medial, 3: lateral, and 4: posterior). Black limb represents prosthetic limb. Each channel was individually controlled with force transducer located under prosthetic foot linked to tactor on thigh. Global reference frame to left with $x-y-z$ corresponding to mediolateralanteroposterior-inferosuperior directions, respectively. 
across the three trials. Raw CoP data were exported and filtered using Visual 3D software (C-motion Inc; Germantown, Maryland) with a low-pass zero-phase lag fourth-order Butterworth filter at a cutoff frequency of 10 Hz. Under each of the standing conditions, Visual 3D was used to calculate two variables of interest (mediolateral and anteroposterior RMS $\left[\mathrm{RMS}_{\mathrm{ML}}\right.$ and $\mathrm{RMS}_{\mathrm{AP}}$, respectively]) (Equation (1)), while a custom program (Visual Basic for Applications, Microsoft Corp; Redmond, Washington) was used to calculate seven variables of interest: PPS of the CoP (Equation (2)), mediolateral and anteroposterior sway amplitude $\left(\mathrm{AMP}_{\mathrm{ML}}\right.$ and $\mathrm{AMP}_{\mathrm{AP}}$, respectively) (Equation (3)), mediolateral and anteroposterior path length $\left(\mathrm{P}_{\mathrm{ML}}\right.$ and $\mathrm{P}_{\mathrm{AP}}$, respectively) (Equation (4)), and mediolateral and anteroposterior velocity ( $\mathrm{MV}_{\mathrm{ML}}$ and $\mathrm{MV}_{\mathrm{AP}}$, respectively) (Equation (5)).

$$
R M S_{\mathrm{AP}}=\sqrt{\frac{y_{1}^{2}+y_{2}^{2} \ldots+y_{n}^{2}}{n}} ; R M S_{\mathrm{ML}}=\sqrt{\frac{x_{1}^{2}+x_{2}^{2} \ldots+x_{n}^{2}}{n}}
$$

where $x$ and $y$ are the respective $x$-direction and $y$-direction coordinate positions of the instantaneous $\mathrm{CoP}$ for consecutive frames and $n$ is the number of frames.

$$
P P S=\frac{f}{n-1} \sum i=1 \text { to } n-1 \sqrt{\left\{\left(x^{i}+1-x^{i}\right)^{2}+\left(y^{i}+1-y^{i}\right)^{2}\right\}},
$$

where $f$ is the sample frequency and $x$ and $y$ are instantaneous CoP coordinate positions.

$$
\left.A M P_{\mathrm{AP}}=\sqrt{\left(y_{\mathrm{A}}-y_{\mathrm{B}}\right)^{2}} ; A M P_{\mathrm{ML}}=\sqrt{\left(x_{\mathrm{A}}-x_{\mathrm{B}}\right.}\right)^{2},
$$

where $x_{\mathrm{A}}, x_{\mathrm{B}}$ and $y_{\mathrm{A}}, y_{\mathrm{B}}$ are the respective maximum and minimum $x$ - and $y$-direction coordinate positions of the instantaneous CoP.

$$
P_{\mathrm{AP}}=y_{1}+y_{2} \ldots+y_{n} ; P_{\mathrm{ML}}=x_{1}+x_{2} \ldots+x_{n},
$$

where $x$ and $y$ are the distances travelled in the $x$ - and $y$ directions between successive frames.

$$
M V_{\mathrm{AP}}=\frac{P_{\mathrm{AP}}}{t} ; M V_{\mathrm{ML}}=\frac{P_{\mathrm{ML}}}{t},
$$

where $t$ is time.

\section{Test Two: Limits of Stability}

The LOS test investigated participants' ability to rapidly shift their center of gravity (CoG) in the anteroposterior and mediolateral directions without stepping or falling. Testing of stability limits was conducted by sequentially highlighting eight targets on a computer screen mounted at eye level at a distance of approximately $1 \mathrm{~m}$. The targets were positioned in a circle at $45^{\circ}$ increments and at a level of difficulty that was 100 percent of the participants' theoretical LOS. The theoretical LOS was based on the participants' height and calculated by the computer software using normative data [44]. The $\mathrm{CoP}$ was displayed in real time on the screen, and the participants were instructed to stand upright and wait for a visual cue to shift their weight sequentially toward each of the eight target positions. Following the visual cue, subjects were to shift their body weight as rapidly as possible from the starting position at the center of the circle to each of the highlighted targets, which were displayed for $8 \mathrm{~s}$ [39]. Of interest in this test was the maximum excursion (ME), directional control (DC), and reaction time (RT). ME was defined as the maximum angular excursion the participants were able to shift their CoG (derived using Equation (6)) in four directions: anterior, posterior, left, and right by calculating the angle of excursion (AE) (Equation (6)). AE was the angle of inclination formed by a line extending from the $\mathrm{CoP}$ and bisecting the CoG and a second line extending vertically from the CoP. The results are expressed as a percentage of the maximum attained angular excursion (expressed in degrees) divided by the theoretical maximum angular excursion for the individual.

$$
\theta=\arcsin \left(\frac{C o P_{y}}{\operatorname{CoG}_{\text {beight }}}\right)-2.30^{\circ},
$$

where $\mathrm{CoP}_{y}$ is the $y$-component of the coordinate position of the instantaneous CoP; $2.30^{\circ}$ refers to the normal forward lean of the angle formed by a line bisecting the CoG and the ankle joints; $C o G_{\text {height }}$ is the distance between CoG and the support surface (z-direction) defined as the height of the individual $\times 0.5527$ [44] .

As the CoG deviated from a central position, a subsequent increase in the magnitude of the angle of excursion 
$(\theta)$ would result. Using the above formula, it was possible to find the ME of this angle of excursion (Equation (7)) by-

$$
M E=\theta_{E}-\theta_{I},
$$

where $\theta_{1}$ is the angle of inclination at trial initiation and $\theta_{\mathrm{E}}$ is the maximum angle of inclination in the intended direction.

DC (Equation (8)) was defined as the total angular distance traveled by the CoG from the central target position expressed as a percentage of the shortest possible distance (a straight line).

$$
\left[\frac{(C o G \text { Path On-Axis })-(C o G \text { Path Off-Axis })}{(C o G \text { Path Off-Axis })}\right] \times 100
$$

A movement in a straight line (CoG path on-axis) with no extraneous movement (CoG path off-axis) would result in a score of 100 percent.

RT was defined as the length of time the participant's CoG took to move from the starting position to a distance of 5 percent of the intended angular distance following the cue to move. For example, if the target distance was $8^{\circ}$, the required threshold would be $0.4^{\circ}$ in the intended direction from the starting position when the cue to move was given.

\section{Test Three: Rhythmic Weight Shift}

The RWS test provided an indication of DC and synchronization of movement. In this test, participants were required to move their $\mathrm{CoG}$, indicated by a cursor on the computer screen. The goal was to shift their body weight, matching as closely as possible the movement of the cursor in two directions: first mediolateral and then anteroposterior. The end point goals (in angular distance [Equation (6)]) were located at $8.00^{\circ}$ in the mediolateral direction and $5.35^{\circ}$ in the anteroposterior direction. For each direction, three trials were performed at three different velocities: slow (3 s between the end points), medium ( $2 \mathrm{~s}$ between the end points), and fast ( $1 \mathrm{~s}$ between the end points). While the target velocity was altered between trials, the displacement of the target remained consistent across testing occasions. This corresponded to three angular velocities (slow, medium, fast) for each direction: 2.67, 4.00, and $8.00 \% \mathrm{~s}$ in the mediolateral direction and 1.78, 2.68, and $5.35 \%$ s in the anteroposterior direction. Altering the velocity is believed to allow one to investigate different aspects of motor control. In the fast velocity, the frequency is of a magnitude similar to gait. As the velocity decreases, so does the required voluntary control of the motion [45].

Data were analyzed using NeuroCom software to identify DC as described by Cheng et al. [46]. This definition uses two variables: (1) on-axis distance: the distance traveled by the moving target and (2) off-axis distance: the actual distance traveled by the CoP. DC (Equation (9)) is defined as the difference of the off-axis distance and the on-axis distance expressed as a percentage of the on-axis distance. It has a similar formula to that used in the LOS test with the only difference that the SD of the CoG path was used in the RWS test.

$$
\left[\frac{(C o G \text { SD On-Axis })-(C o G \text { SD Off-Axis })}{(C o G \text { SD On-Axis })}\right] \times 100 .
$$

On-axis velocity (AV) (Equation (10)) was also investigated and was defined as the participants' ability to match the velocity of the moving target. This considers only the velocity in the intended direction and not the deviations from the intended direction.

$$
\frac{\text { Total Angular Displacement in Intended Direction }}{\text { Total Length of Time }}
$$

All tests were conducted on one occasion. Before testing, participants were fitted with a safety harness suspended above the support surface. It was adjusted to ensure that participants only received support in the case of a fall. Throughout testing, participants were instructed to stand on the force plate with the lateral border of their shoes aligned with one of three marks on the surface of the plate. The appropriate line was determined by the height of the participant, per the manufacturer's recommendations [44].

The position of the force transducer under the prosthetic foot was determined on an individual basis. Position for the heel transducer was the same for all feet and was positioned at the bisected mediolateral width of the foot, approximately $2 \mathrm{~cm}$ from the posterior edge of the foot. Anterior, medial, and lateral positions were determined 
depending on the design of the prosthetic foot. Optimal position was determined by positioning the transducers under the hard, flat surface analogous to "metatarsal heads" in an anatomical foot. The anterior transducer was at midwidth position just anterior to the metatarsal head position, and the medial and lateral transducers, respectively, at the most medial and lateral border of the foot in a position located slightly posterior to the anterior tactor. Small adjustments were made in a limited number of cases in which the foot design prevented the transducers from producing adequate signal for operation.

Vibrating tactors linked to the force transducers were positioned at the measured midpoint of the thigh on the affected side. This position was determined on an individual basis by measuring the linear distance from the greater trochanter to the lateral femoral condyle and placing the markers at the intersection of the proximal and distal segments. The tactors were then placed in a horizontal configuration perpendicular to the longitudinal (axial) axis of the thigh. A single tactor was placed in four separate locations corresponding to $0^{\circ}, 90^{\circ}, 180^{\circ}$, and $270^{\circ}$. A spatial resolution of $90^{\circ}$ has been shown to be as effective as higher resolutions at conveying bodytilt information [27]. Anterior direction was designated as $0^{\circ}$, with $90^{\circ}$ and $270^{\circ}$ designated medial and lateral and $180^{\circ}$ located posteriorly. Anterior was determined by having the participant stand comfortably and locating the anterior surface of the thigh. In this way, anterior was analogous with the line of progression of the body and not of the limb itself.

Following the placement of the tactors, individual tactor tuning was conducted. During this time, participants were requested to stand in a comfortable, upright position that was considered the "neutral" position. The aim of the tuning was to ensure that minimal vibration was felt when standing in the neutral position and that the vibration sensation increased when the force transducers under the foot were loaded. The cutoff threshold was adjusted for each of the four individual force transducers to minimize the amount of multiple tactor vibration in the neutral position. By requesting that the participants shift their CoG in the four sensor directions utilizing movement from the ankle, we conducted optimization of the cutoff threshold. The gain used in all testing was $130 \mathrm{~mA}$ RMS, which translates into a displacement amplitude for the tactors of $0.16 \mathrm{~mm}$ at the threshold for activation. The cutoff threshold for vibration was uniform for all participants, and the tuning simply adjusted the signal magnitude required from the force transducers in order to initiate vibration. After vibration was initiated, it increased proportionately based on the magnitude of the signal from the relevant force transducer.

A period of approximately $20 \mathrm{~min}$ was taken to set the cutoff threshold for all the tactors. This was followed by a period of $10 \mathrm{~min}$ in which the participants were encouraged to shift their CoG in the mediolateral and anteroposterior directions and to familiarize themselves with the function of the device. Participants then moved to the force platform and the complete test protocol was conducted to allow familiarization. The participants were instructed to stand comfortably with arms by their sides maintaining an upright posture and to shift their CoG using as little hip motion as possible. Under all practice sessions, the feedback unit was in operation.

Following the practice session, the testing protocol was conducted under two randomly selected conditions: VIB or NOVIB. A period of rest was offered after each individual test, and a 10 min rest was required for all participants after completing half the protocol. The approximate time to complete the protocol was 50-60 min. The participants were never required to stand longer than $7 \mathrm{~min}$ and, because of the structure of the tests, were never focused on the balance task for longer than $20 \mathrm{~s}$ at a time.

\section{Data Analysis}

Statistical analysis was performed using PASW Statistics 18.0.1 (IBM; Armonk, New York). Results of a Kolmogorov-Smirnov test established no violations of normality in the data. SB was analyzed using a three-way multivariate analysis of variance (MANOVA) with three independent variables (vibration, support-surface, vision) and nine dependent variables RMS $_{M L}, \mathrm{RMS}_{\mathrm{AP}}$, PPS, $\left.\mathrm{AMP}_{\mathrm{ML}}, \mathrm{AMP}_{\mathrm{AP}}, \mathrm{P}_{\mathrm{ML}}, \mathrm{P}_{\mathrm{AP}}, \mathrm{MV}_{\mathrm{ML}}, \mathrm{MV}_{\mathrm{AP}}\right)$. LOS test was analyzed using a two-way MANOVA with two independent variables (vibration, direction) and three dependent variables (RT, ME, DC). RWS was analyzed using a three-way MANOVA with three independent variables (vibration, direction, velocity) and two dependent variables (AV, DC). Confidence intervals of the mean differences are given for main effect (VIB-NOVIB) for each of the SB, LOS, and RWS tests and interaction effects where present. Statistical significance was determined using a critical alpha level of 0.05 for all primary analyses. Where the MANOVA resulted in a significant main effect for VIB-NOVIB, a univariate analysis of variance was conducted to identify significantly different dependent variables with a Bonferroni adjustment for multiple comparisons. 


\section{RESULTS}

\section{Standing Balance}

All 24 subjects completed the four conditions. Six falls were recorded among three participants (five falls in condition 4, one fall in condition 3). Because of these falls, two subjects had insufficient trials to allow a mean value to be calculated. For these two subjects, the remaining successful trial was used for analysis.

There was a statistically significant main effect (VIB-NOVIB) (Table 2). Post-hoc analysis identified $\mathrm{AMP}_{\mathrm{ML}}$ as the only significantly different variable $(p=$ 0.001 ), indicating increased $\mathrm{AMP}_{\mathrm{ML}}$ in the VIB condition. No significant interaction effect was seen between the remaining independent variables (vibration $\times$ vision, $p>0.99$; vibration $\times$ surface, $p=0.13$; vibration $\times$ vision $\times$ surface, $p=0.65)$.

\section{Limits of Stability}

No falls were recorded during the LOS test. The results showed a statistically significant main effect (VIB-NOVIB) for the independent variable RT ( $p=$ 0.01 ). The RT was faster with vibration (Table 3). No significant interaction effect was present for the remaining variable (vibration $\times$ direction, $p=0.66$ ).

\section{Rhythmic Weight Shift}

No falls were recorded during the RWS test. The results showed no statistically significant main effect (VIB-NOVIB) $(p=0.89)$, and no significant interaction effect was seen with the remaining variables (AV: vibration $\times$ direction, $p=0.34$; vibration $\times$ velocity, $p=0.95$; vibration $\times$ direction $\times$ velocity, $p=0.63$; DC: vibration $\times$ direction, $p=0.43$; vibration $\times$ velocity, $p=0.25$; vibration $\times$ direction $\times$ velocity, $p=0.29$ ) (Table 4).

Table 2.

Mean and standard deviation values for standing balance results for each condition. Area to right shows result of the main effect analysis (vibration [VIB]-no vibration [NOVIB]) with mean difference (Diff), 95\% confidence interval (CI), and p-values for post hoc analysis. Variables included path length per second (PPS), mean mediolateral and anteroposterior path length $\left(\mathrm{P}_{\mathrm{ML}}\right.$ and $\left.\mathrm{P}_{\mathrm{AP}}\right)$, mediolateral and anteroposterior sway amplitude ( $\mathrm{AMP}_{\mathrm{ML}}$ and $\left.\mathrm{AMP}_{\mathrm{AP}}\right)$, mean mediolateral and anteroposterior velocity ( $\mathrm{MV}_{\mathrm{ML}}$ and $\mathrm{MV}_{\mathrm{AP}}$ ), and mediolateral and anteroposterior rootmean-square $\left(\mathrm{RMS}_{\mathrm{ML}}\right.$ and $\left.\mathrm{RMS}_{\mathrm{AP}}\right)$.

\begin{tabular}{|c|c|c|c|c|c|c|c|}
\hline \multirow[b]{2}{*}{ Variable } & \multicolumn{4}{|c|}{ Condition } & \multicolumn{3}{|c|}{ Main Effect "Vibration" } \\
\hline & 1 & 2 & 3 & 4 & Diff & $\begin{array}{c}95 \% \text { CI } \\
\text { (VIB-NOVIB) }\end{array}$ & $\begin{array}{c}p \text { - } \\
\text { Value }\end{array}$ \\
\hline$\overline{\text { PPS VIB (cm/s) }}$ & $1.08 \pm 0.46$ & $1.71 \pm 0.62$ & $1.86 \pm 0.91$ & $3.34 \pm 1.12$ & 0.06 & $-0.17-0.30$ & 0.59 \\
\hline PPS NOVIB (cm/s) & $0.99 \pm 0.35$ & $1.61 \pm 0.52$ & $1.60 \pm 0.56$ & $3.54 \pm 1.39$ & & & \\
\hline $\mathrm{P}_{\mathrm{ML}} \mathrm{VIB}(\mathrm{cm})$ & $8.67 \pm 5.61$ & $12.46 \pm 6.84$ & $14.22 \pm 9.22$ & $23.89 \pm 10.45$ & 0.70 & $-1.68-3.08$ & 0.56 \\
\hline $\mathrm{P}_{\mathrm{ML}} \operatorname{NOVIB}(\mathrm{cm})$ & $6.72 \pm 3.28$ & $10.43 \pm 3.63$ & $11.17 \pm 4.54$ & $28.11 \pm 15.55$ & & & \\
\hline $\mathrm{AMP}_{\mathrm{ML}} \mathrm{VIB}(\mathrm{cm})$ & $2.04 \pm 1.18$ & $2.86 \pm 1.68$ & $4.45 \pm 3.29$ & $4.91 \pm 3.41$ & 1.01 & $0.41-1.60$ & 0.001 \\
\hline $\mathrm{AMP}_{\mathrm{ML}}$ NOVIB (cm) & $1.62 \pm 0.94$ & $2.18 \pm 1.03$ & $2.85 \pm 1.59$ & $3.59 \pm 1.94$ & & & \\
\hline $\mathrm{MV}_{\mathrm{ML}} \mathrm{VIB}(\mathrm{cm} / \mathrm{s})$ & $0.43 \pm 0.28$ & $0.62 \pm 0.34$ & $0.71 \pm 0.46$ & $1.19 \pm 0.52$ & 0.04 & $-0.08-0.15$ & 0.56 \\
\hline $\mathrm{MV}_{\mathrm{ML}} \operatorname{NOVIB}(\mathrm{cm} / \mathrm{s})$ & $0.34 \pm 0.16$ & $0.52 \pm 0.18$ & $0.56 \pm 0.23$ & $1.40 \pm 0.78$ & & & \\
\hline $\mathrm{RMS}_{\mathrm{ML}} \mathrm{VIB}(\mathrm{cm})$ & $1.17 \pm 0.78$ & $1.30 \pm 0.87$ & $1.29 \pm 0.99$ & $1.51 \pm 0.92$ & 0.06 & $-0.18-0.30$ & 0.63 \\
\hline $\mathrm{RMS}_{\mathrm{ML}}$ NOVIB (cm) & $0.92 \pm 0.68$ & $1.19 \pm 0.77$ & $1.22 \pm 0.80$ & $1.70 \pm 0.94$ & & & \\
\hline $\mathrm{P}_{\mathrm{AP}} \mathrm{VIB}(\mathrm{cm})$ & $17.95 \pm 6.43$ & $29.20 \pm 9.36$ & $31.02 \pm 13.83$ & $57.21 \pm 18.39$ & 0.93 & $-2.67-4.53$ & 0.61 \\
\hline $\mathrm{P}_{\mathrm{AP}} \operatorname{NOVIB}(\mathrm{cm})$ & $17.12 \pm 6.24$ & $28.69 \pm 9.81$ & $27.41 \pm 10.02$ & $50.18 \pm 19.57$ & & & \\
\hline $\mathrm{AMP}_{\mathrm{AP}} \mathrm{VIB}(\mathrm{cm})$ & $2.56 \pm 1.71$ & $3.57 \pm 2.26$ & $4.03 \pm 2.44$ & $5.06 \pm 2.80$ & 0.42 & $-0.24-1.08$ & 0.21 \\
\hline $\mathrm{AMP}_{\mathrm{AP}}$ NOVIB (cm) & $2.16 \pm 1.85$ & $2.70 \pm 1.66$ & $3.90 \pm 2.50$ & $4.80 \pm 2.97$ & & & \\
\hline $\mathrm{MV}_{\mathrm{AP}} \mathrm{VIB}(\mathrm{cm} / \mathrm{s})$ & $0.89 \pm 0.32$ & $1.46 \pm 0.47$ & $1.55 \pm 0.69$ & $2.86 \pm 0.92$ & 0.05 & $-0.13-0.23$ & 0.61 \\
\hline $\mathrm{MV}_{\mathrm{AP}} \operatorname{NOVIB}(\mathrm{cm} / \mathrm{s})$ & $0.86 \pm 0.31$ & $1.43 \pm 0.49$ & $1.37 \pm 0.50$ & $2.92 \pm 0.98$ & & & \\
\hline $\mathrm{RMS}_{\mathrm{AP}} \mathrm{VIB}(\mathrm{cm})$ & $5.20 \pm 1.58$ & $5.41 \pm 1.71$ & $5.22 \pm 1.74$ & $5.49 \pm 1.67$ & -0.08 & $-0.56-0.41$ & 0.76 \\
\hline $\mathrm{RMS}_{\mathrm{AP}}$ NOVIB $(\mathrm{cm})$ & $5.26 \pm 1.72$ & $5.49 \pm 1.71$ & $5.17 \pm 1.77$ & $5.71 \pm 1.62$ & & & \\
\hline
\end{tabular}


Table 3.

Mean and standard deviation (SD) for main effect limits of stability analysis, mean difference (Diff) of vibration (VIB)-no vibration (NOVIB) trials, 95\% confidence interval (CI), and $p$-values for post hoc analysis. Variables included reaction time (RT), maximum excursion (ME), and directional control (DC).

\begin{tabular}{|c|c|c|c|c|}
\hline Variable & Mean \pm SD & Diff & 95\% CI (VIB-NOVIB) & $p$-Value \\
\hline ME VIB (\%) & $73.3 \pm 16.8$ & -1.00 & $-4.9-2.9$ & 0.62 \\
\hline DC VIB (\%) & $80.6 \pm 8.6$ & 1.99 & $-0.5-4.5$ & 0.12 \\
\hline DC NOVIB (\%) & $78.6 \pm 10.6$ & & & \\
\hline
\end{tabular}

Table 4.

Mean and standard deviation (SD) for rhythmic weight shift composite score results for vibration (VIB)-no vibration (NOVIB) trials, 95\% confidence interval (CI), and $p$-values for post hoc analysis. Variables included mediolateral and anteroposterior on-axis velocity (AV $\mathrm{ML}_{\mathrm{L}}$ and $\left.\mathrm{AV}_{\mathrm{AP}}\right)$ and mediolateral and anteroposterior directional control ( $\mathrm{DC}_{\mathrm{ML}}$ and $\left.\mathrm{DC}_{\mathrm{AP}}\right)$.

\begin{tabular}{|c|c|c|c|c|}
\hline Variable & Mean \pm SD & Difference & $\begin{array}{c}95 \% \text { CI } \\
\text { (VIB-NOVIB) }\end{array}$ & $p$-Value \\
\hline $\mathrm{AV}_{\mathrm{ML}}$ NOVIB & $5.0 \pm 2.5$ & & & \\
\hline $\mathrm{AV}_{\mathrm{AP}}$ NOVIB & $3.3 \pm 1.6$ & & & \\
\hline $\mathrm{DC}_{\mathrm{ML}} \mathrm{VIB}$ & $82 \pm 7.0$ & -0.7 & $-2.8-1.4$ & 0.51 \\
\hline $\mathrm{DC}_{\mathrm{AP}}$ NOVIB & $75 \pm 8.4$ & & & \\
\hline
\end{tabular}

\section{DISCUSSION}

The aim of this study was to evaluate the effects of a vibratory feedback system on static and dynamic balance in persons with unilateral transtibial limb loss. Our experimental hypothesis was disproven because the results indicate no consistent pattern of benefit or hindrance with the use of feedback on any test of postural stability. The performance of SB indicated an increased amplitude of excursion for the $\mathrm{CoP}$ in the mediolateral direction $\left(\mathrm{AMP}_{\mathrm{ML}}\right)$ with the addition of vibratory feedback. No other variables in SB were found to be significantly different. In LOS, RT was significantly reduced with the addition of the vibratory feedback, suggesting participants responded faster.

The observed increase in $\mathrm{AMP}_{\mathrm{ML}}$ found in the $\mathrm{SB}$ test under the VIB condition is in contrast with previous research [26-28] that showed improved performance in a sample of persons with reduced vestibular function. Increases in traditional sway parameters (velocity and displacement) are linked to delayed use of closed-loop (feedback) mechanisms of postural control [47]. Because of this, the addition of vibration would have been expected to reduce the magnitudes of these variables if prosthesis users were able to use the feedback in this closed-loop system. This was not the case in the current investigation and suggests that the prosthesis users were unable to make use of the additional feedback. The magnitude of the differences (mean \pm SD: $101 \pm 1.88$, Table 2) is comparable with those known to differentiate older individuals at risk of falling from those at low risk of falling [11]. However, because none of the other eight variables showed any statistically significant differences in the SB analysis, the result should be viewed with caution. The $\mathrm{AMP}_{\mathrm{ML}}$ has no published data specifically related to prosthetic users but is a measure of the greatest deviation between two points in a given uniplanar direction ( $x$ or $y$ ). The participants had no significant difference in the $\mathrm{MV}_{\mathrm{ML}}$ yet they had larger deviations, as indicated by $\mathrm{AMP}_{\mathrm{ML}}$. This suggests that even though they had greater magnitude of deviations, they had none of the dangerous high-velocity movements associated with increased fall risk [12]. This could be the result of the participants voluntarily shifting their body weight in an effort to elicit 
vibration from the tactors without increasing $\mathrm{MV}_{\mathrm{ML}}$ and $\mathrm{RMS}_{\mathrm{ML}}$, which are linked to increased risk of falling [11-12]. This could indicate that the participants were adopting an increasingly exploratory postural strategy in SB when the device was active. When regulation of postural stability must be precise, small movements of the CoG within safe margins are permitted in an effort to use feedforward mechanisms of postural control to update the central nervous system on body orientation [48]. For this reason, the increased $\mathrm{AMP}_{\mathrm{ML}}$ measures should not be deemed negative results. Instead, this may reflect a shift of dominant control strategies, from feedback to feedforward as a result of the experimental intervention. Given the link between increases in CoP velocity and fall injuries [12], the results of the SB test suggest that the addition of vibration is unlikely to affect the incidence of falls or fall-related injuries. This is evident by the magnitude of differences between the VIB and NOVIB conditions, which remained small regardless of the variable.

In the LOS test, RT decreased in the VIB conditions. This is of interest because it shows that the participants were able to coordinate a directed movement of the body's CoG faster with the addition of vibratory feedback. However, this outcome only evaluates the participants' time to react and not the overall time to reach the angular goal, the accuracy of the motion toward the target, or whether the participants overshoot the target. In this sense, reacting to the cue in the LOS is perhaps an easier task than the SB test, which requires constant modification during $20 \mathrm{~s}$. In the "wait-to-respond" scenario in the LOS test, participants are possibly using a preemptive feedforward mechanism in response to the task and not a feedback mechanism [48-50]. This preemptive feedforward mechanism has previously been identified during postural tasks in transtibial prosthesis users [51-52] and indicates a sensorimotor adaptation that makes repetitive or predictable motor tasks more efficient. The RT test is representative of this type of predictable motor task in which the participant can make anticipatory sensorimotor adaptations in advance of the task. This current study is the first to suggest that a system providing vibratory feedback that is proportional to the force magnitude under the prosthetic foot may stimulate the use of an anticipatory feedforward mechanism of postural control among persons with lower-limb amputations. The results showed that the participants' response was more than $1 / 10$ of a second faster with the addition of vibratory feedback (VIB mean \pm SD: $0.87 \pm 0.29$ s; NOVIB mean \pm SD:
$0.980 \pm 0.330 \mathrm{~s}$; mean \pm SD difference: $0.113 \pm 0.310 \mathrm{~s}$; Table 3). The results are similar to those reported by Trueblood et al. [53] among community-living elderly fallers and non-fallers (non-fallers mean \pm SD: $0.86 \pm$ $0.39 \mathrm{~s}$; fallers mean \pm SD: $0.98 \pm 0.38 \mathrm{~s}$ ), which means the improvement in RT for the prosthesis users is potentially clinically relevant. Because electromyography (EMG) onset latencies of as little as 350 to $500 \mathrm{~ms}$ are of adequate magnitude to be within voluntary control [54], the magnitude of difference in RTs in this study $(1,130 \mathrm{~ms})$ suggests the participants were having a portion of the improvements from these voluntary (conscious) control mechanisms. Without EMG data collected simultaneously, it is impossible to determine which proportion of this improvement is coming from nonvoluntary control of postural response $(<350 \mathrm{~ms})$ and which is coming from voluntary control (>350 ms) [54]. Yet, the overall response showed a clear improvement with VIB values. They also clearly indicate that transtibial prosthesis users in this study have delayed RTs compared with nondisabled, matched age groups as described by Nolan et al. [55]. Using averaged composite scores for the age groups of 40-49 yr and 50-59 yr, the composite RT reported in Nolan et al. is $0.62 \pm 0.25 \mathrm{~s}$, which indicates that our participants performed worse regardless of feedback condition (VIB mean \pm SD: $0.87 \pm 0.29$ s; NOVIB mean \pm SD: $0.98 \pm 0.33 \mathrm{~s}$ ). Although not part of the original research question, upon further investigation, the largest improvements clearly came in reactions toward the prosthetic limb and toward the anterior direction. Further research is warranted to investigate this apparent directionally dependent interaction. With the known challenges prosthesis users face in the anteroposterior direction, this improvement could be of particular relevance for this sample group.

The possibility that the participants were more attentive to the RT task because of audible sound from the tactors must also be considered. In quiet standing when the participants' CoP was near the "neutral zone," there was very little audible sound from the tactor. To hear any vibration required complete silence in the room and focused listening from the participant. Because of a constant ambient noise from the laboratory equipment, it is unlikely the tactor sound would have been perceptible to the participants. As the cue for motion was provided visually on the computer screen, any additional auditory stimulus (and attempt to focus on it) would most likely have been distracting and not beneficial. 
The decision to provide predetermined stance widths based on the subjects' heights, as per the manufacturer's recommendations, has associated consequences. The LOS and RWS tests specify the particular widths as part of their protocol. Yet, in the case of the SB test, we could have allowed the individuals to select their foot position. Kirby et al. showed a significant increase between mediolateral CoP displacement when participants' feet were together than when they were separated [56]. Though for stance widths from 15 to $45 \mathrm{~cm}$, there was no continued decrease in CoP displacement as stance width increased. Because the participants in the current study had stance widths of this magnitude, influence on the results is likely to be minimal.

Provision of a so-called "neutral zone," whereby the participants could stand comfortably while receiving very little feedback, may also have presented limitations. If, in the tuning phase, we had allowed an overlap of the cutoff thresholds so multiple tactors were operating simultaneously, additional feedback might have been provided to the participants. Additionally, knowing when the tactors were activated and their duration may have provided additional insight not available in the current study. Duclos et al. showed that the introduction of vibratory stimuli to neck and hip musculature influenced postural symmetry, as indicated by increased symmetrical loading of the prosthesis and that this had a "posteffect" of at least 13 min [30]. We did not look at postural symmetry in this investigation, yet one may question whether this posteffect had any significant effect on the prosthetic users. The vibratory stimulus in the current investigation was randomized, and periods of no vibration never exceeded $13 \mathrm{~min}$. If a posteffect were present, one would expect an influence on the NOVIB trials interspersed randomly between the VIB trials, with the result being no significant difference between the two conditions. We found a significant difference between VIB and NOVIB conditions, indicating no presence of a posteffect. This could also be due to the differences in the vibratory stimulus used in the current investigation and in Duclos et al. [30] (frequency 230 vs $80 \mathrm{~Hz}$; amplitude 0.16 vs $0.50 \mathrm{~mm}$ ). One form of subsensory vibration, referred to as "stochastic resonance," has also been shown to improve balance in many populations [57]. Stochastic resonance functions in biomedical applications by enhancing the function of biological systems, such as sensation, with the application of a random noise signal at subsensory levels. The current study differs from studies of stochas- tic resonance in two ways. The stimulus signal in this study was not random in nature but a sine wave (tone bursts at $250 \mathrm{~Hz}$ ) and was above the level required for conscious perception. For this reason, we believe the phenomenon observed to be the result of consciously perceived vibratory feedback and not the result of transient vibratory stimulus.

Although Sienko et al. showed that CoP results mirror the trunk-tilt results in a sample of persons with vestibular loss [27], prosthesis users lack a physiological ankle and are unable to utilize the so-called ankle strategy for postural stability on the prosthetic side $[52,58]$. An inability to use the ankle strategy on the prosthetic side means that prosthesis users rely more on a hip strategy for stability, including increased shear forces at the support surface [59]. As the force transducers for the feedback system in this study only respond to normal forces, the shear forces resulting from the hip strategy would not register. Future research incorporating shear forces into the feedback system is subsequently of interest. Additionally, prosthetic users tend to have larger deviations of the CoP under the intact limb than the prosthetic limb in standing [20]. As motion of the CoP from the force-platform represents the physiological response to motion of the CoM from both sides of the body [60], a limitation of this study is that the feedback device only provided feedback proportional to the pressure shifts under the prosthetic side. It would be beneficial in future studies to use two force platforms to identify the contributions of the intact and prosthetic sides individually. This information would provide additional insight into how the feedback is integrated into the sensorimotor response of the participants. We do believe, however, that any reduced motion of the CoP under the prosthetic foot was controlled for by the operation of the tactor controller. During the tuning phase, the minimum threshold for tactor operation and amplification gain were optimized to give as large a window of operation as possible. During pilot testing, it became evident that, because of the compliance of the intact foot, the participants could not apply adequate pressures to individual tactors in order to elicit a vibration on that side. Because the prosthetic feet were relatively stiff, placement of tactors on the prosthetic side resulted in greater transmission of forces to the tactors. Seinko et al. also used an array encompassing a greater number of tactors than the current investigation [27]. Future research should address these limitations and 
identify to what extent they influence the function of a feedback device on transtibial prosthesis users.

Given that the two variables found to be significantly different in this study (AMP $_{M L}$ in SB test and RT in LOS test) may be influenced by feedforward mechanisms of postural control, further investigation in this area would be prudent. Utilizing additional methods, such as frequency analysis in SB and EMG during rapid motor tasks, it would be possible to further explore how a simple feedback device could positively affect the multiple postural control mechanisms in transtibial prosthesis users.

\section{CONCLUSIONS}

The use of a vibratory feedback system on static and dynamic balance for persons using unilateral transtibial prostheses produced no consistent pattern of benefit or hindrance with the use of feedback, with statistically significant differences in SB and LOS but not RWS. Performance in SB resulted in greater deviations of the CoP in the mediolateral direction. Performance in the LOS test indicated faster RTs. The results suggest the use of vibratory feedback may specifically benefit the feedforward (open-loop) control mechanisms of postural control for transtibial prosthesis users.

\section{ACKNOWLEDGMENTS}

\author{
Author Contributions: \\ Study concept and design: D. Rusaw, K. Hagberg, L. Nolan, \\ N. Ramstrand. \\ Acquisition of data: D. Rusaw. \\ Analysis and interpretation of data: D. Rusaw, K. Hagberg, \\ L. Nolan, N. Ramstrand. \\ Drafting of manuscript: D. Rusaw, K. Hagberg, L. Nolan, \\ N. Ramstrand. \\ Critical revision of manuscript for important intellectual content: \\ D. Rusaw, K. Hagberg, L. Nolan, N. Ramstrand. \\ Obtained funding: D. Rusaw, N. Ramstrand.
}

Financial Disclosures: The authors have declared that no competing interests exist.

Funding/Support: This material was based on work supported by the Swedish Association of Prosthetists and Orthotists and Innovationsbron. Additional Contributions: The authors wish to thank Kjell-Åke Nilsson, Jessica Crafoord, Maria Glemne, and Prof. Björn Rydevik for their assistance in this study.

Institutional Review: Participants provided written consent and were advised that they may discontinue their participation at any time. Ethical approval for the study was granted by the Ethical Regional Review Board in Linköping, Sweden.
Participant Follow-Up: The authors do not plan to inform participants of the publication of this study.

\section{REFERENCES}

1. Nielsen JB, Sinkjaer T. Afferent feedback in the control of human gait. J Electromyogr Kinesiol. 2002;12(3):213-17. [PMID:12086816] http://dx.doi.org/10.1016/S1050-6411(02)00023-8

2. MacKay-Lyons M. Central pattern generation of locomotion: a review of the evidence. Phys Ther. 2002;82(1):69-83. [PMID:11784280]

3. van Velzen JM, van Bennekom CA, Polomski W, Slootman JR, van der Woude LH, Houdijk H. Physical capacity and walking ability after lower limb amputation: a systematic review. Clin Rehabil. 2006;20(11):999-1016.

[PMID:17065543]

http://dx.doi.org/10.1177/0269215506070700

4. Miller WC, Speechley M, Deathe B. The prevalence and risk factors of falling and fear of falling among lower extremity amputees. Arch Phys Med Rehabil. 2001; 82(8):1031-37. [PMID:11494181]

http://dx.doi.org/10.1053/apmr.2001.24295

5. Miller WC, Deathe AB. A prospective study examining balance confidence among individuals with lower limb amputation. Disabil Rehabil. 2004;26(14-15):875-81.

[PMID:15497916]

http://dx.doi.org/10.1080/09638280410001708887

6. Miller WC, Deathe AB, Speechley M, Koval J. The influence of falling, fear of falling, and balance confidence on prosthetic mobility and social activity among individuals with a lower extremity amputation. Arch Phys Med Rehabil. 2001;82(9):1238-44. [PMID:11552197] http://dx.doi.org/10.1053/apmr.2001.25079

7. Kavounoudias A, Tremblay C, Gravel D, Iancu A, Forget R. Bilateral changes in somatosensory sensibility after unilateral below-knee amputation. Arch Phys Med Rehabil. 2005;86(4):633-40. [PMID:15827911] http://dx.doi.org/10.1016/j.apmr.2004.10.030

8. Quai TM, Brauer SG, Nitz JC. Somatosensation, circulation and stance balance in elderly dysvascular transtibial amputees. Clin Rehabil. 2005;19(6):668-76.

[PMID:16180604]

http://dx.doi.org/10.1191/0269215505cr857oa

9. Courtemanche R, Teasdale N, Boucher P, Fleury M, Lajoie Y, Bard C. Gait problems in diabetic neuropathic patients. Arch Phys Med Rehabil. 1996;77(9):849-55.

[PMID:8822673] http://dx.doi.org/10.1016/S0003-9993(96)90269-5

10. Buatois S, Gueguen R, Gauchard GC, Benetos A, Perrin PP. Posturography and risk of recurrent falls in healthy 
non-institutionalized persons aged over 65. Gerontology. 2006;52(6):345-52. [PMID:16905886]

http://dx.doi.org/10.1159/000094983

11. Norris JA, Marsh AP, Smith IJ, Kohut RI, Miller ME. Ability of static and statistical mechanics posturographic measures to distinguish between age and fall risk. J Biomech. 2005;38(6):1263-72. [PMID:15863111] http://dx.doi.org/10.1016/j.jbiomech.2004.06.014

12. Piirtola M, Era P. Force platform measurements as predictors of falls among older people-a review. Gerontology. 2006;52(1):1-16. [PMID:16439819] http://dx.doi.org/10.1159/000089820

13. Raymakers JA, Samson MM, Verhaar HJ. The assessment of body sway and the choice of the stability parameter(s). Gait Posture. 2005;21(1):48-58. [PMID:15536033] http://dx.doi.org/10.1016/j.gaitpost.2003.11.006

14. Buckley JG, O'Driscoll D, Bennett SJ. Postural sway and active balance performance in highly active lower-limb amputees. Am J Phys Med Rehabil. 2002;81(1):13-20. [PMID:11807327] http://dx.doi.org/10.1097/00002060-200201000-00004

15. Geurts AC, Mulder TW, Nienhuis B, Rijken RA. Dual-task assessment of reorganization of postural control in persons with lower limb amputation. Arch Phys Med Rehabil. 1991;72(13):1059-64. [PMID:1741657]

16. Hermodsson Y, Ekdahl C, Persson BM, Roxendal G. Standing balance in trans-tibial amputees following vascular disease or trauma: a comparative study with healthy subjects. Prosthet Orthot Int. 1994;18(3):150-58. [PMID:7724348]

17. Vrieling AH, van Keeken HG, Schoppen T, Otten E, Hof AL, Halbertsma JP, Postema K. Balance control on a moving platform in unilateral lower limb amputees. Gait Posture. 2008;28(2):222-28. [PMID:18207407] http://dx.doi.org/10.1016/j.gaitpost.2007.12.002

18. Winter DA, Prince F, Frank JS, Powell C, Zabjek KF. Unified theory regarding $\mathrm{A} / \mathrm{P}$ and $\mathrm{M} / \mathrm{L}$ balance in quiet stance. J Neurophysiol. 1996;75(6):2334-43. [PMID:8793746]

19. Gage WH, Winter DA, Frank JS, Adkin AL. Kinematic and kinetic validity of the inverted pendulum model in quiet standing. Gait Posture. 2004;19(2):124-32.

[PMID:15013500] http://dx.doi.org/10.1016/S0966-6362(03)00037-7

20. Rougier PR, Bergeau J. Biomechanical analysis of postural control of persons with transtibial or transfemoral amputation. Am J Phys Med Rehabil. 2009;88(11):896-903. [PMID:19661773] http://dx.doi.org/10.1097/PHM.0b013e3181b331af

21. Postema K, Hermens HJ, de Vries J, Koopman HF, Eisma WH. Energy storage and release of prosthetic feet. Part 1: biomechanical analysis related to user benefits. Prosthet Orthot Int. 1997;21(1):17-27. [PMID:9141122]
22. Rietman JS, Postema K, Geertzen JH. Gait analysis in prosthetics: opinions, ideas and conclusions. Prosthet Orthot Int. 2002;26(1):50-57. [PMID:12043926] http://dx.doi.org/10.1080/03093640208726621

23. Nederhand MJ, Van Asseldonk EH, van der Kooij H, Rietman HS. Dynamic Balance Control (DBC) in lower leg amputee subjects; contribution of the regulatory activity of the prosthesis side. Clin Biomech (Bristol, Avon). 2012;27(1): 40-45. [PMID:21889241] http://dx.doi.org/10.1016/j.clinbiomech.2011.07.008

24. Nielsen DH, Shurr DG, Golden JC, Meier K. Comparison of energy cost and gait efficiency during ambulation in below-knee amputees using different prosthetic feet-a preliminary report. J Prosthet Orthot. 1988;1(1):24-31. http://dx.doi.org/10.1097/00008526-198810000-00006

25. Hafner BJ, Sanders JE, Czerniecki J, Fergason J. Energy storage and return prostheses: does patient perception correlate with biomechanical analysis? Clin Biomech (Bristol, Avon). 2002;17(5):325-44. [PMID:12084537] http://dx.doi.org/10.1016/S0268-0033(02)00020-7

26. Peterka RJ, Wall C 3rd, Kentala E. Determining the effectiveness of a vibrotactile balance prosthesis. J Vestib Res. 2006;16(1-2):45-56. [PMID:16917168]

27. Sienko KH, Balkwill MD, Oddsson LI, Wall C. Effects of multi-directional vibrotactile feedback on vestibular-deficient postural performance during continuous multi-directional support surface perturbations. J Vestib Res. 2008; 18(5-6):273-85. [PMID:19542601]

28. Wall C 3rd, Weinberg MS. Balance prostheses for postural control. IEEE Eng Med Biol Mag. 2003;22(2):84-90.

[PMID:12733464] http://dx.doi.org/10.1109/MEMB.2003.1195701

29. Sabolich JA, Ortega GM. Sense of feel for lower-limb amputees: a phase-one study. J Prosthet Orthot. 1994;6(2): 36-41. http://dx.doi.org/10.1097/00008526-199400620-00003

30. Duclos C, Roll R, Kavounoudias A, Roll JP, Forget R. Vibration-induced post-effects: a means to improve postural asymmetry in lower leg amputees? Gait Posture. 2007;26(4):595-602. [PMID:17236772] http://dx.doi.org/10.1016/j.gaitpost.2006.12.005

31. Fan RE, Culjat MO, King CH, Franco ML, Boryk R, Bisley JW, Dutson E, Grundfest WS. A haptic feedback system for lower-limb prostheses. IEEE Trans Neural Syst Rehabil Eng. 2008;16(3):270-77. [PMID:18586606] http://dx.doi.org/10.1109/TNSRE.2008.920075

32. Fan RE, Culjat MO, King CH, Franco ML, Sedrak M, Bisley JW, Dutson EP, Grundfest WS. A prototype haptic feedback system for lower-limb prostheses and sensory neuropathy. Stud Health Technol Inform. 2008;132:115-19. [PMID:18391269]

33. Hafner BJ. Clinical prescription and use of prosthetic foot and ankle mechanisms: a review of the literature. J Prosthet 
Orthot. 2005;17(4S):5-11.

http://dx.doi.org/10.1097/00008526-200510001-00004

34. Persson BM, Liedberg E. A clinical standard of stump measurement and classification in lower limb amputees. Prosthet Orthot Int. 1983;7(1):17-24. [PMID:6856447]

35. della Volpe R, Popa T, Ginanneschi F, Spidalieri R, Mazzocchio R, Rossi A. Changes in coordination of postural control during dynamic stance in chronic low back pain patients. Gait Posture. 2006;24(3):349-55.

[PMID:16311036]

http://dx.doi.org/10.1016/j.gaitpost.2005.10.009

36. Shumway-Cook A, Hutchinson S, Kartin D, Price R, Woollacott $\mathrm{M}$. Effect of balance training on recovery of stability in children with cerebral palsy. Dev Med Child Neurol. 2003;45(9):591-602. [PMID:12948326] http://dx.doi.org/10.1111/j.1469-8749.2003.tb00963.x

37. Chaudhry H, Bukiet B, Ji Z, Findley T. Measurement of balance in computer posturography: comparison of methods-a brief review. J Bodyw Mov Ther. 2011;15(1):82-91. [PMID:21147423] http://dx.doi.org/10.1016/j.jbmt.2008.03.003

38. Rose J, Wolff DR, Jones VK, Bloch DA, Oehlert JW, Gamble JG. Postural balance in children with cerebral palsy. Dev Med Child Neurol. 2002;44(1):58-63. [PMID:11811652] http://dx.doi.org/10.1017/S0012162201001669

39. Hageman PA, Leibowitz JM, Blanke D. Age and gender effects on postural control measures. Arch Phys Med Rehabil. 1995;76(10):961-65. [PMID:7487439] http://dx.doi.org/10.1016/S0003-9993(95)80075-1

40. Brouwer B, Culham EG, Liston RA, Grant T. Normal variability of postural measures: implications for the reliability of relative balance performance outcomes. Scand J Rehabil Med. 1998;30(3):131-37. [PMID:9782539] http://dx.doi.org/10.1080/003655098444048

41. O’brien RM. Generalizability coefficients are reliability coefficients. Quality \& Quantity. 1995;29(4):421-28. http://dx.doi.org/10.1007/BF01106066

42. Dodd K, Hill K, Haas R, Luke C, Millard S. Retest reliability of dynamic balance during standing in older people after surgical treatment of hip fracture. Physiother Res Int. 2003;8(2):93-100. [PMID:12879731] http://dx.doi.org/10.1002/pri.276

43. Clark S, Riley MA. Multisensory information for postural control: sway-referencing gain shapes center of pressure variability and temporal dynamics. Exp Brain Res. 2007;176(2):299-310. [PMID:16874512] http://dx.doi.org/10.1007/s00221-006-0620-6

44. Neurocom International. Balance manager systems: clinical operations guide. Clackamas (OR): Neurocom International; 2008. p. 9.
45. Liao HF, Mao PJ, Hwang AW. Test-retest reliability of balance tests in children with cerebral palsy. Dev Med Child Neurol. 2001;43(3):180-86. [PMID:11263688]

46. Cheng P-T, Wang CM, Chung CY, Chen CL. Effects of visual feedback rhythmic weight-shift training on hemiplegic stroke patients. Clin Rehabil. 2004;18(7):747-53. [PMID:15573830] http://dx.doi.org/10.1191/0269215504cr778oa

47. Collins JJ, De Luca CJ, Burrows A, Lipsitz LA. Agerelated changes in open-loop and closed-loop postural control mechanisms. Exp Brain Res. 1995;104(3):480-92.

[PMID:7589299] http://dx.doi.org/10.1007/BF00231982

48. Gatev P, Thomas S, Kepple T, Hallett M. Feedforward ankle strategy of balance during quiet stance in adults. J Physiol. 1999;514(Pt 3):915-28. [PMID:9882761] http://dx.doi.org/10.1111/j.1469-7793.1999.915ad.x

49. Collins JJ, De Luca CJ. Open-loop and closed-loop control of posture: a random-walk analysis of center-of-pressure trajectories. Exp Brain Res. 1993;95(2):308-18.

[PMID:8224055] http://dx.doi.org/10.1007/BF00229788

50. Pavol MJ, Pai YC. Feedforward adaptations are used to compensate for a potential loss of balance. Exp Brain Res. 2002;145(4):528-38. [PMID:12172665] http://dx.doi.org/10.1007/s00221-002-1143-4

51. Aruin AS, Nicholas JJ, Latash ML. Anticipatory postural adjustments during standing in below-the-knee amputees. Clin Biomech (Bristol, Avon). 1997;12(1):52-59.

[PMID:11415672] http://dx.doi.org/10.1016/S0268-0033(96)00053-8

52. Viton JM, Mouchnino L, Mille ML, Cincera M, Delarque A, Pedotti A, Bardot A, Massion J. Equilibrium and movement control strategies in trans-tibial amputees. Prosthet Orthot Int. 2000;24(2):108-16. [PMID:11061197] http://dx.doi.org/10.1080/03093640008726533

53. Trueblood PR, Hodson-Chennault N, McCubbin A, Youngclarke D. Performance and impairment-based assessments among community dwelling elderly: sensitivity and specificity. Issues on Aging. 2001;24(1):2-6.

54. Carpenter MG, Allum JH, Honegger F. Directional sensitivity of stretch reflexes and balance corrections for normal subjects in the roll and pitch planes. Exp Brain Res. 1999; 129(1):93-113. [PMID:10550507] http://dx.doi.org/10.1007/s002210050940

55. Nolan M, Nitz J, Choy NL, Illing S. Age-related changes in musculoskeletal function, balance and mobility measures in men aged 30-80 years. Aging Male. 2010;13(3): 194-201. http://dx.doi.org/10.3109/13685531003657818

56. Kirby RL, Price NA, MacLeod DA. The influence of foot position on standing balance. J Biomech. 1987;20(4):423-27. 


\section{[PMID:3597457]}

http://dx.doi.org/10.1016/0021-9290(87)90049-2

57. McDonnell MD, Abbott D. What is stochastic resonance? Definitions, misconceptions, debates, and its relevance to biology. PLOS Comput Biol. 2009;5(5):e1000348. [PMID:19562010] http://dx.doi.org/10.1371/journal.pcbi.1000348

58. Vanicek N, Strike S, McNaughton L, Polman R. Postural responses to dynamic perturbations in amputee fallers versus nonfallers: a comparative study with able-bodied subjects. Arch Phys Med Rehabil. 2009;90(6):1018-25. [PMID:19480879]

http://dx.doi.org/10.1016/j.apmr.2008.12.024

59. Horak FB, Nashner LM. Central programming of postural movements: adaptation to altered support-surface configurations. J Neurophysiol. 1986;55(6):1369-81.

[PMID:3734861]
60. Winter DA. Biomechanics and motor control of human movement, 4th ed. Hoboken (NJ): John Wiley \& Sons, Inc; 2009. p. 370.

Submitted for publication May 13, 2011. Accepted in revised form January 27, 2012.

This article and any supplementary material should be cited as follows:

Rusaw D, Hagberg K, Nolan L, Ramstrand N. Can vibratory feedback be used to improve postural stability in persons with transtibial limb loss? J Rehabil Res Dev. 2012;49(8):1239-54.

http://dx.doi.org/10.1682/JRRD.2011.05.0088

\begin{tabular}{|c|c|}
\hline $\begin{array}{l}\text { nu subussions screened Br: } \\
\text { iThenticate }\end{array}$ & Crossef НЕнве \\
\hline 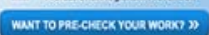 & $\begin{array}{l}\text { CROSSREF.ORG } \\
\text { THE CITATION LIHKIHG BACKEONE }\end{array}$ \\
\hline
\end{tabular}


